\title{
New Techniques to Address Asymmetry in 3D Structure Analysis of Helical Biofilaments Imaged by Cryo-EM
}

\author{
Charles Sindelar, Garrett Debs and Andrew Huehn \\ Yale University, New Haven, Connecticut, United States
}

Filamentous, helical assemblies are integral to the function of a vast swath of biomolecular processes. By taking advantage of the inherent symmetry of helical objects, many such systems are now routinely characterized at near atomic resolution by cryo-EM using established 3D structural refinement methods. However, a rising challenge in this area is to address filamentous systems where symmetry is broken. Important examples include filaments irregularly decorated with binding partners (such as motor proteins, branching factors, or regulatory proteins) and tubular filaments that substantially deform from a helically symmetric shape via flexing/breathing. In such cases, 3D structures of relevant molecular components may be drastically compromised or may not even be achievable at all.

Here, I describe a strategy developed in my group to tackle this class of problems, incorporating a novel algorithm to regularize filament subunit trajectories contaminated by missing or erroneously defined 'outlier' subunit coordinates. The strategy can be divided into three steps: (1) conventional helical structure refinement using previously established methods (IHRSR (E. Egelman, 2010; E. H. Egelman, 2000)) to obtain an initial 3D model describing the average structure of all filaments, as well as estimates of the positions/orientations of all imaged filament subunits; (2) 'Smoothing' of filament trajectories by the new algorithm to obtain a self-consistent model of each imaged filament, described as a continuously connected, multi-subunit object; (3) focused 3D structure refinement and/or classification of filament subunits (individually or in small groups) to account for variability in composition and/or position/orientation in localized regions of the filaments. Following initial helical model generation (step (1)), multiple iterations of steps (2) and (3) are performed until convergence is achieved, resulting in a description of the position and composition of each subunit within each imaged filament that accounts for deviations from symmetry. Application of this strategy enables a more complete structural analysis of a diverse set of asymmetric, filamentous assemblies.

\section{References}

Egelman, E. H. (2000). A robust algorithm for the reconstruction of helical filaments using single-particle methods. Ultramicroscopy, 85 (4), 225-234. 